\section{Probiotic L. gasseri strain (LG21) for the upper gastrointestinal tract acting through improvement of indigenous microbiota}

To cite: Koga Y, Ohtsu T, Kimura $\mathrm{K}$, et al. Probiotic L. gasseri strain (LG21) for the upper gastrointestinal tract acting through improvement of indigenous microbiota. BMJ Open Gastro 2019;6:e000314. doi:10.1136/ bmjgast-2019-000314

Received 24 May 2019 Revised 29 June 2019 Accepted 16 July 2019
Check for updates

(c) Author(s) (or their employer(s)) 2019. Re-use permitted under CC BY-NC. No commercial re-use. See rights and permissions. Published by BMJ.

${ }^{1}$ Gastroenterology, Tokai University School of Medicine, Isehara City, Japan

${ }^{2}$ Food Microbiology Research Laboratories, Meiji, Hachiouji City, Japan

Correspondence to Yasuhiro Koga; jpn.probio1998@mbr.nifty.com

\section{ABSTRACT}

Objective To describe probiotics including a Lactobacillus gasseri strain LG21 used for the upper gastrointestinal tract, which are considered to act through improvement of indigenous microbiota inhabiting there.

Background and design Because the early definition of probiotics emphasized their effects on improving the intestinal microbial ecology, their effects on the intestinal tract and its immunity have been considered common general benefits associated with probiotics. This conclusion was also based on a body of successful clinical trials whose endpoints were the prevention or treatment of intestinal diseases. In contrast to intestinal microbiota, our understanding of the role of gastric microbiota in human health and physiology remains poor, as the bacterial load in the stomach is considered too small to exert a significant effect due to the highly acidic environment of the human stomach. Therefore, the intervention using probiotics in the stomach is still limited at present.Results:In this article using representative 38 quoted articles, we first describe the gastric microbiota, as the indigenous microbiota in the stomach is thought to be significantly involved in the pathophysiology of this organ, since probiotics exert their beneficial effects through improving the resident microbiota. We then review the present status and future prospects of probiotics for the treatment of upper gastrointestinal diseases by quoting representative published articles, including our basic and clinical data.

Conclusions Probiotics have been demonstrated to suppress Helicobacter pylori in the stomach, and are also expected to improve functional dyspepsia through the correction of dysbiotic gastric microbiota.

\section{INTRODUCTION}

In 1989, Fuller ${ }^{1}$ defined probiotics as 'a live microbial feed supplement that beneficially affects the host animal by improving its intestinal microbial balance'. This influential definition was then followed by the standard definition presented by the Joint (Food and Agriculture Organizationns of the UNs) FAO/ WHO Expert Consultation in 2001'? 'a live microorganism that, when administered in adequate amounts, confers a health benefit on the host'. The International Scientific Association for Probiotics and Prebiotics consensus statements recently reported in $2014^{3}$ also retained the main body of these definitions.

The gut contains a complex and dynamic microbial ecosystem with a high density of bacteria whose cell number can reach as high as approximately $10^{12} / \mathrm{g}$ faeces, the total number of which is thus estimated to be 10-fold larger than the total number of eukaryotic cells in the human body. ${ }^{4}$ Because the early definition of probiotics emphasised their effects on improving the intestinal microbial ecology, their effects on the intestinal tract and its immunity have been considered common general benefits associated with probiotics. This conclusion was also based on a body of successful clinical trials whose endpoints were the prevention or treatment of intestinal diseases, such as infectious diarrhoea, antimicrobial-associated diarrhoea, constipation, irritable bowel syndrome (IBS), inflammatory bowel diseases, necrotising enterocolitis and symptomatic abdominal pain.

By contrast, the size of the gastric microbial mass, in which probiotic bacteria are considered to exert their beneficial effects, has been thought to be very small because of the high acidity and frequent peristalsis to the gut in the stomach. The high acidity due to the secreted gastric acid kills probiotic bacteria as well as gastric commensal bacteria. Thus the application of probiotics to the stomach or proximal small intestine has historically been considered impractical. However, the Maastricht 2-2000 Consensus Report ${ }^{5}$ first noted the possible role of gastric probiotics in the treatment of Helicobacter pylori infection in the stomach, as several studies reported that some probiotic bacteria exerted an inhibitory effect on $H$. pylori in vitro and in vivo. 
Nevertheless, even at present the use of probiotics for the treatment of illness in the upper gastrointestinal (GI) tract, including the oesophagus, stomach and duodenum, remains limited and far rather than their application in the colon.

In this article, we first describe the gastric microbiota, as the indigenous microbiota in the stomach is thought to be significantly involved in the pathophysiology of this organ, since probiotics exert their beneficial effects through improving the resident microbiota. We then review the present status and future prospects of probiotics for the treatment of upper GI diseases by quoting representative published articles, including our basic and clinical data.

\section{GASTRIC MICROBIOTA}

\section{Gastric microbiota analyses by 16S rRNA gene profiling} using high-throughput DNA sequencing

So far, in reports of gastric microbiota analyses using traditional culturing methods, the bacterial count was so small $\left(\sim 10^{3}\right.$ colony-forming unit $(\mathrm{CFU}) / \mathrm{mL}$ gastric fluid $(\mathrm{GF}))$ that it was almost impossible to accurately determine an overview of the bacterial community structure in the stomach. ${ }^{6}$

In 2006, Bik et $a l^{7}$ performed a 16S rRNA gene analysis using 1833 sequences obtained from 23 human gastric endoscopic biopsy samples and identified 128 bacterial phylotypes. Li et al also performed 16S rRNA gene profiling using 1223 non- $H$. pylori sequences from 10 gastric biopsy samples, which were classified into 133 phylotypes. In both of those studies regarding the gastric mucosa-associated microbiota using high-throughput $16 \mathrm{~S}$ rDNA sequencing, the results were so similar, although the two studies analysed racially distinct populations living in different countries. Both studies found two predominantly abundant genera-Streptococcus and Prevotella - that accounted for approximately half of the total detected species. In 2015, Tsuda et at performed a meta-16S analysis of the gastric luminal microbiota from Japanese subjects with far greater sequencing depth. They obtained roughly 40000 high-quality reads for the sequence analysis from $45 \mathrm{GF}$ samples and identified that those two genera were also the most prevalent ones, accounting for approximately $50 \%$ of the total species in the stomach. In addition, Neisseria and Rothia were also ranked among the top 5 most prevalent bacteria in those three reports, including the mucosa-associated and luminal microbiota studies. This similarity in the bacterial composition between the two intragastric subpopulations (mucosa-associated and luminal) suggested that the luminal bacteria colonise the mucosa and the mucosal bacteria flow back into the lumen. H. pylori is also considered to colonise the stomach in that manner, although this bacterium exclusively inhabits the mucosa, including gastric epithelial cells and the surface mucous layer. ${ }^{10}$ Indeed, H. pylori was identified as the major bacterial species in the gastric mucosal specimens, ${ }^{11}$ but as a relatively small-size population in the GF from subjects with $H$. pylori infection.

\section{Difference in the microbial community structure among GF, saliva and faeces}

Tsuda et at compared three bacterial communities in the oral cavity, stomach and colon along the alimentary tract using 45 subjects, including 18 patients taking protonpump inhibitors (PPIs). Stimulated saliva, GF and faeces were obtained from each subject for the microbiota analysis using 16S rRNA gene sequencing method. A total of 3000 high-quality reads for the sequence analysis were randomly selected from each sample and used for the subsequent bioinformatic analyses.

No marked difference in the degree of bacterial species richness ( $\alpha$-diversity) was found among salivary, GF and faecal microbiotas. The average numbers of operational taxonomic units, that is, the number of bacterial species, all ranged from 120 to $140 / \mathrm{mL}$ or $\mathrm{g}$ in the three microbiota samples, indicating that the overall species richness was so similar among these microbiotas, regardless of differences in their habitats. In contrast, the log CFU bacterial counts (median) quantified by real-time PCR were $8.7 / \mathrm{mL}, 7.8 / \mathrm{mL}$ and $10.5 / \mathrm{g}$ in the saliva, GF and faeces, respectively, meaning that the total bacterial count was sensitive to different environmental factors such as the $\mathrm{pH}$, oxygen concentration and nutrient availability. It is also noteworthy that the copy number of bacterial genome is far higher than the CFU number of bacteria in the GF $\left(10^{7.8} / \mathrm{mL}\right.$ vs $\left.10^{2 \sim 4} / \mathrm{mL}\right)$, suggesting that more than $99.9 \%$ of the GF bacteria are dead and/or viable but non-culturable. These results suggest that the diversity and mass size of GF microbiota might be great enough to significantly affect the pathophysiology of the stomach through the metabolites and components of the bacteria.

The structure of the whole bacterial community was compared using unique fraction metric (UniFrac) analyses. Unweighted and weighted UniFrac principal coordinate analyses showed that the salivary and GF samples aggregated to form a common cluster different from the cluster of faecal samples (figure 1). This result suggested that the majority of GF microbiota might originate from microbes in the oral cavity, possibly through the ingestion of the saliva and masticated foods into the stomach. In contrast, the cluster of faecal microbiota was totally different from that of GF microbiota, despite the fact that the latter constantly flow downstream into the former, indicating a large difference in the microbial ecosystem between the stomach and intestine.

The analysis at the genus level using the OTUs generated from the 16S rRNA gene reads also showed high similarity between the salivary and GF microbiota. Indeed, the five most prevalent genera were (in descending order) Streptococcus, Prevotella, Neisseria, Rothia and Veillonella, and Streptococcus, Prevotella, Actinomyces, Neisseria and Rothia in the salivary and GF microbiotas, respectively. Of note, these observations markedly differed from those of faecal microbiota, where the five most prevalent genera were 

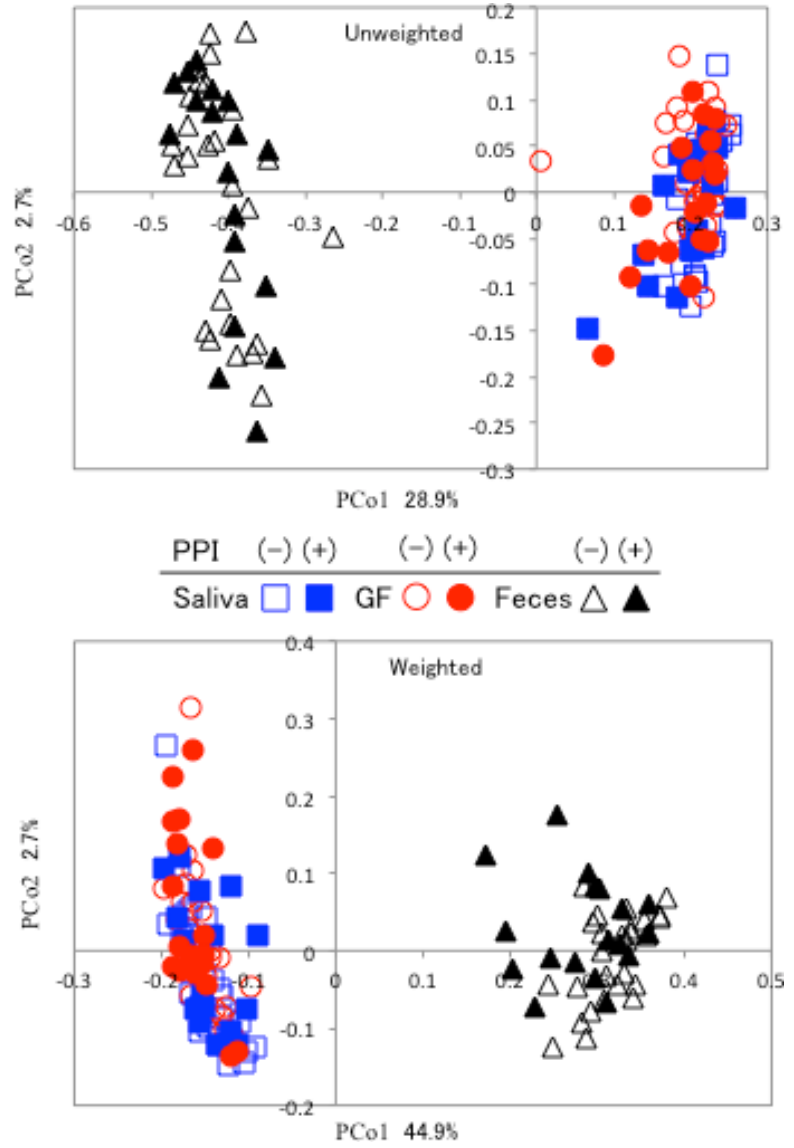

Figure 1 Result of UniFrac analysis of three microbiota communities. The overall bacterial community was demonstrated by unweighted and weighted UniFrac principal coordinate analyses. Squares, circles and triangles indicate the salivary, GF and faecal samples, respectively. Open and filled symbols represent PPI non-users and PPI users, respectively. Cited with permission from ref ${ }^{9}$. GF, gastric fluid; PPI, proton-pump inhibitor; PCo 1, 2, Principal coordinate 1, 2

\section{Bifidobacterium, Bacteroides, Ruminococcus, Faecalibacterium and Clostridium in the faecal microbiota.}

\section{Influence of gastric acidity on the gastric microbiota}

The stomach is a harsh environment for many micro-organisms because strong gastric acid kills many ingested microbes. The peak acidity of the GF is as low as $\mathrm{pH}$ $1-2$, which is quite reasonable, as a major physiological role of the gastric acid is to kill exogenous pathogenic bacteria that migrate to the digestive tract through the mouth. Due to the high acidity, the number of culturable bacteria is at most approximately $1000 \mathrm{CFU} / \mathrm{mL}$ in the GF when sampled in the morning after overnight fasting. ${ }^{12}$ Because of this very small number of bacteria in the culture-based method, analyses of the bacterial composition in the stomach have been limited to only a several bacterial genera like Streptococcus and Lactobacillus.

Since the 1970s and 1980s, highly effective antiacid drugs such as histamine 2 receptor antagonists and PPIs, respectively, have been widely used around the world as therapy for GI diseases caused by excessive gastric acids. These drugs, especially PPIs, are so efficient in suppressing the gastric acid secretion that the $\mathrm{pH}$ value of the GF reaches almost neutral (approximately 7.0) in many of PPI users. However, in response to such weakened acidity, the number of live bacteria in the GF increases dramatically. Indeed, Ruddell $e t a l^{3}$ reported that the $\mathrm{pH}$ value and the average number of bacteria in the GF changed from 2.6 to 6.0 and $10^{1.8}$ to $10^{4.6} \mathrm{CFU} / \mathrm{mL}$, respectively, in patients with peptic ulcer being treated with antacid drugs for 1 month. It was also reported that GF with $\mathrm{pH}<4$ has bactericidal activity, whereas GF with $\mathrm{pH}>4$ enables bacteria alive in the stomach. ${ }^{14}$ Tsuda et a $\ell^{\rho}$ similarly reported a significant correlation between the acidity and the number of live bacteria in the GF using 45 subjects, including PPI users. These findings support the notion that the stomach is a potential site that can be inhabited by many bacteria, but strong gastric acid inhibits the colonisation of those bacteria there when analysed by traditional culture-based method.

\section{Biological role of indigenous gastric microbiota}

In humans, the number of detectable indigenous bacteria in the stomach is very small when culture-based methods are used. However, in the stomach of mice bred in a conventional environment, the number of such bacteria, predominantly consisting of lactobacilli, was quite high at around $10^{7} \mathrm{CFU} / \mathrm{g}$ mucosa. ${ }^{15}$ The lower acidity ( $\mathrm{pH}$ 4-5) in the stomach of mice than in humans is thought to enable lactobacilli to colonise the stomach.

Takahashi et $a l^{16}$ examined the biological role of such lactobacilli in the stomach using a gnotobiotic murine system. They found that the Lactobacillus-associated gnotobiotic mice had a dramatically decreased expression of the gastrin gene in comparison with germ-free mice. Gastrin is predominantly produced in the $\mathrm{G}$ cells of the gastric antrum and stimulates the acid-secreting parietal cells. ${ }^{17}$ Consequently, gastric acid secretion was also decreased in the mice colonised by lactobacilli when compared with germ-free mice. In addition, an increase in the expression of the genes related to the muscle layer development, such as nebulin and troponin genes, was observed in the Lactobacillus-associated mice. The muscle layer is responsible for gastric motility. Weak or abnormal motility of the stomach due to poor development of the muscle layer might lead to impaired gastric emptying, such as that developing during functional dyspepsia (FD) ${ }^{18}$ Furthermore, the infection of germ-free mice with $H$. pylori also induced the downregulation and upregulation of gastrin and muscle genes, respectively. ${ }^{16}$ Saito $e t a l^{19}$ further reported that the muscle layer of the stomach was significantly thickened in mice infected with $H$. pylori.

These reports suggested that both the indigenous gastric microbiota including lactobacilli and exogenous $H$. pylori significantly affect the regulation of gastric acid secretion and the development of gastric motility. Given that the prevalence of $H$. pylori infection is now very low in developed countries, large numbers of people in these countries might be considered to have 'germ-free' 
stomachs with high acidity and poor motility, despite being free from the pathogenicity of $H$. pylori.

\section{USE OF PROBIOTICS FOR UPPER GI DISORDERS Development of a probiotic Lactobacillus strain for the suppression of $\boldsymbol{H}$. pylori in human}

To establish an animal model of a $H$. pylori-infected host, we tried to infect mice with $H$. pylori by oral inoculation in the late 1990s. ${ }^{15}$ However, no infection was obtained when we used mice in a specific pathogen-free (SPF) environment. Successful infection was only obtained when mice were kept in a germ-free environment. To determine why $H$. pylori could not colonise the stomach of SPF mice, we examined the bacterial population in the stomach of SPF mice by the culture-based method. We found that a large number of lactobacilli were colonising the stomach of SPF mice (more than $10^{8} \mathrm{CFU} / \mathrm{g}$ in the stomach tissue). To confirm that those indigenous lactobacilli actually protect the stomach from $H$. pylori infection, we then established several kinds of gnotobiotic mice by inoculating different bacterial species into the stomach of germ-free mice. We then tried to infect these gnotobiotic mice with $H$. pylori. As a result, we again found that lactobacilli-associated gnotobiotic mice did not become infected with $H$. pylori, although germ-free mice easily became infected. In contrast, the gnotobiotic mice colonised by the bacteria other than lactobacilli such as Enterococcus faecalis or Staphylococcus aureus were easily infected with $H$. pylori. These results suggested that the genus Lactobacillus has a stronger ability to inhibit $H$. pylori infection in the stomach than other genera, suggesting this genus is recommended for use as a probiotic strain in case of $H$. pylori infection.

Based on the results of our basic study, we screened more than 200 strains of lactobacilli to select one that exhibited a higher level of resistance to acidity and binding ability to human gastric epithelium. Then we finally identified the strain LG21 (Lactobacillus gasseri OLL2716) as the most suitable for trials in humans. Indeed, LG21 at the stationary growth phase can survive even in the culture broth with a $\mathrm{pH}$ of 2.5 , which is almost the same value as the $\mathrm{pH}$ value of gastric acid. Sasaki ${ }^{20}$ reported that the LG21 strain had several defence mechanisms enabling it to withstand acid stress, including the upregulation of the genes of cation ATP-binding cassette (ABC) transporter and arginine/ornithine antiporter and the downregulation of the genes of transcription and protein synthesis. The acid stress response may be naturally indispensable in lactobacilli, as their growth and activity are always accompanied by the production of a large amount of lactic acid, which causes acidification of the external environment, leading to the arrest of their growth and possible cell death. The LG21 strain is thus thought to be a highly efficient Lactobacillus strain capable of surviving acidic injury.

In a coculture system for an adherence assay, the number of LG21 strain required to suppress the binding of H. pylori to MKN45, a human gastric epithelial cell line, as well as decrease the production of proinflammatory interleukin-8 (IL-8) from MKN45 was 100-fold less than that of inputted $H$. pylori. In contrast, inactivated and dead LG21 failed to exert any suppressive effect whatsoever on binding or IL-8 production. These results underscored the importance of both surviving strong acid exposure and achieving adhesion to the gastric mucosa for the probiotics to work well in the human stomach.

To assess the efficacy of LG21 as a probiotic for $H$. pylori infection in humans, first, $31 \mathrm{H}$. pylori-infected subjects ingested yoghourt containing $10^{9}$ CFU LG21 every day for 8 weeks. ${ }^{21}$ The ${ }^{13} \mathrm{C}$-urea breath test results and serum pepsinogens I/II measurement were significantly improved after LG21 treatment. LG21 was thus shown to be effective in reducing both the $H$. pylori load and gastric mucosal inflammation. However, none of the subjects achieved complete eradication of $H$. pylori at 3 months after the termination of LG21 treatment. Most other studies using probiotics alone also showed a reduction in $H$. pylori colonisation and an improvement in $H$. pylori-induced gastritis. ${ }^{22}$ However, none demonstrated the complete eradication of $H$. pylori infection. Nevertheless, the long-term intake of probiotics may have a beneficial effect on $H$. pylori-infected people by reducing the risk of developing disorders associated with a high degree of gastric inflammation. Such treatment using probiotics was also reported to effectively prevent children from incurring primary $H$. pylori infection. ${ }^{23}$ In addition, clinical trials regarding the effect of supplementation with probiotics including LG21 demonstrated a significant effect both on increasing eradication rate and alleviating the side effects by antimicrobials. ${ }^{24} 25$

Gastric microbial community associated with the occurrence of gastric cancers

The infection of gastric mucosa with $H$. pylori progresses through the stages of chronic mucosal gastritis, atrophy and intestinal metaplasia before carcinoma develops. However, eradication of $H$. pylori does not always result in the complete prevention of gastric cancer in the subjects who have already suffered such gastric mucosal pathological changes. Recently, a microbiota profiling analysis revealed that the gastric microbiota in the stomach suffering from carcinoma was characterised by reduced microbial diversity, a decreased abundance of $H$. pylori and the enrichment of other bacterial genera, mostly represented by intestinal commensals. ${ }^{26}$ These results suggested that the change in the gastric microbiota composition might exert a significant effect on the development of gastric carcinogenesis, although $H$. pylori infection plays a critical role in the initiation of gastric carcinoma. Given that both dysbiosis and reduced acidity in the stomach can be improved by probiotics, the intake of gastric probiotics like the LG21 strain might be useful for preventing the development of carcinogenesis even after the eradication of $H$. pylori. Indeed, LG21, which secretes lactic acid, increased the $\alpha$-diversity of the gastric microbiota in the human stomach (unpublished data, 
Igarashi, M., et al. 2017), and lactic acid has been proven to alter the microbiota composition. ${ }^{27}$

\section{USE FOR FUNCTIONAL DYSPEPSIA}

\section{Possible involvement of dysbiotic gastric microbiota in FD}

FD is a major clinical issue affecting patients' well-being as it is highly prevalent globally and is associated with recurrent symptoms, which consist of postprandial distress syndrome (PDS) and/or epigastric pain syndrome (EPS) that are unexplained after a routine clinical evaluation. ${ }^{28}$ While the exact pathophysiology of FD remains to be clarified, gastric motility disturbance, such as delayed gastric emptying and impaired gastric accommodation after meals, and visceral hypersensitivity have been postulated as critical underlying mechanisms. ${ }^{29}$ Although there is a considerable amount of evidence to suggest that dysbiosis of the intestinal microbiota is involved in the pathophysiology of IBS, a functional gastrointestinal disorder (FGID) originating from the intestine, little is known about the gastric microbiota on their role in the pathophysiology of FD, an FGID originating from the stomach and possibly the proximal small intestine.

While antimicrobial therapy targeting $H$. pylori has been reported to be effective in treating FD-like symptoms in some patients, the improving effect on the symptoms might not be mediated by the eradication of $H$. pylori but by the effect of antimicrobials on the other bacteria. Indeed, Miwa $e t a l^{30}$ reported that cure of $H$. pylori infection did not improve symptoms in $H$. pylori-infected patients with FD-like symptoms in a double-blind, placebo-controlled clinical study. Recently it was reported that the oral administration of the antimicrobial rifaximin to patients with FD led to adequate relief of global PDS, implying the possible involvement dysbiosis in the pathogenesis of FD. ${ }^{31}$

Furthermore in our study to examine the effect of the probiotic strain LG21 on FD-like symptoms associated with $H$. pylori infection, the severity of PDS was significantly lower after LG21 treatment than before the treatment, although no significant difference was found in the laboratory tests indicating the number and activity of $H$. pylori colonising the stomach, such as the H. pylori stool antigen density and urea breath test. ${ }^{32}$ As the normalisation of dysbiotic microbiota is one of the dominant effects of probiotics, such improvement in the FD-like symptoms by LG21 in patients infected with $H$. pylori might be associated with a change in the gastric indigenous bacterial community other than $\mathrm{H}$. pylori. These results urged us to investigate both the effect of probiotics on patients with FD without $H$. pylori infection and the role of gastric microbiota in the pathophysiology underlying FD.

\section{Effect of LG21 on patients suffering from FD}

Ohtsu et $a l^{33}$ evaluated the efficacy of LG21 treatment on patients with FD without $H$. pylori infection through a double-blind, placebo controlled, randomised clinical trial (RCT). Patients were randomly assigned to ingest active yoghourt containing $10^{9} \mathrm{CFU}$ of LG21 or placebo yoghourt containing no LG21 for 12 weeks. One hundred and six subjects (mean age $42.8 \pm 9.0$ years old) completed the trial. According to questionnaire analysis in the trial, the impressions regarding the overall effect on gastric symptoms were more positive in the LG21 group than in the placebo group (statistical trend; $\mathrm{p}=0.073$ ). Furthermore, the elimination rates for major FD symptoms were $35.3 \%$ and $17.3 \%$ in the LG21 and placebo groups, respectively $(p=0.048)$. These results indicated that LG21 had a beneficial effect on FD without $H$. pylori involvement. Regarding the differential effect on PDS or EPS, LG21 had a greater beneficial effect on PDS than on the EPS symptoms. Because prokinetic agents improving GI motility have been reported to have beneficial effect on PDS, ${ }^{34}$ the therapeutic effect of LG21 might be focused mainly on gastric motility abnormalities causing PDS.

In conclusion, this study is the first involving a probiotic treatment trial with an RCT design, strict definition of FD and use of sufficiently reliable outcomes, although the sample size was relatively small for the assessment of a comprehensive participant-reported outcome as an endpoint.

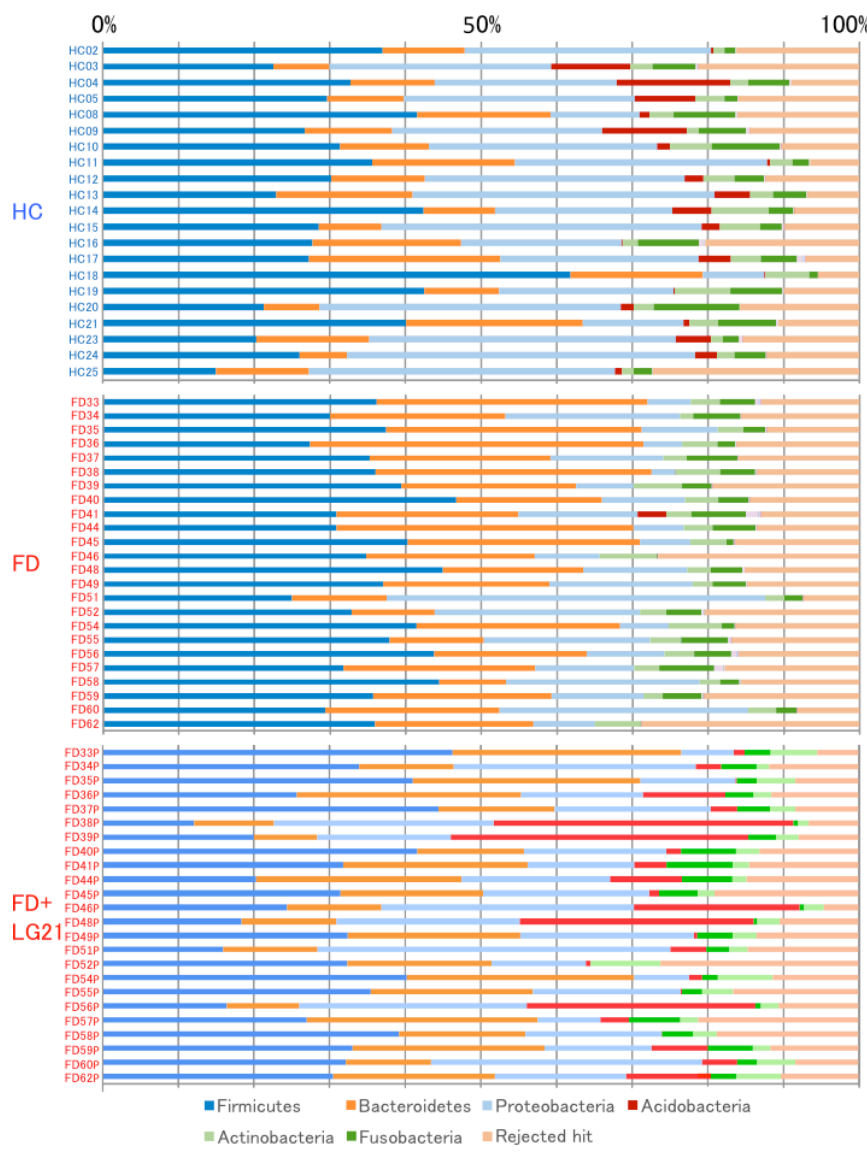

Figure 2 Bacterial composition at the phylum level. The relative abundance of the bacterial composition at the phylum level in each sample from the subjects in the HC, FD and FD after LG21 yoghourt treatment groups is shown on a bar chart. Cited with permission from ref ${ }^{35}$. FD, functional dyspepsia; HC, healthy control; LG21, Lactobacillus gasseri OLL2716. 
Change in the gastric microbiota and its restoration by LG21 in patients with $\mathrm{FD}$

Although the probiotic strain LG21 was demonstrated to exert therapeutic effect on FD, possibly due to improvements in the GI motility abnormality, the exact underlying mechanism of the action remained largely unknown. Therefore, we comparatively analysed the GF microbiota between patients with FD and healthy controls (HCs) in order to assess the effect of LG21 on the microbiota for the reason mentioned in the 'Possible involvement of dysbiotic gastric microbiota in FD' section. ${ }^{35}$

Twenty-four Japanese patients with FD who met the Rome III definition and 21 age-matched and gendermatched HC volunteers were enrolled. The GF was sampled using a nasogastric tube after an overnight fast. In the patients with FD, the sampling of GF was done twice (before and after the treatment with LG21). For the microbiota analysis, the V3-V4 region of the 16S rRNA gene was amplified using bacterial DNA from the GF, and then about 30000 high-quality amplicons per sample were grouped into operational OTUs.
In the bacterial composition analysis at the phylum level, the GF microbiota had a Bacteroidetes $>$ Proteobacteria and Bacteroidetes $<$ Proteobacteria abundances in the FD and HC groups, respectively, while the most dominant phylum was Firmicutes in both groups (figure 2). To investigate the mechanism underlying such a marked difference in the microbiota between patients with FD and HCs, we examined the similarity in the frequency of identification among samples in the clustering of genera (figure 3, bottom side). As a result, two distinct clusters of genera were found. One cluster was formed by a particular group consisting of 32 genera, including typical inhabitants of the intestine such as Bifidobacterium, Faecalibacterium and Bacteroides. The other cluster was formed by 51 genera, including typical inhabitants of the stomach of healthy individuals, such as Neisseria, Prevotella and Streptococcus. Of note, the 32 intestinal-type genera were particularly dominant in subjects with FD but scarce in HC subjects. Given that the ratio of GF samples in which the bile acid was detectable was significantly greater in the FD group than in the HC group in

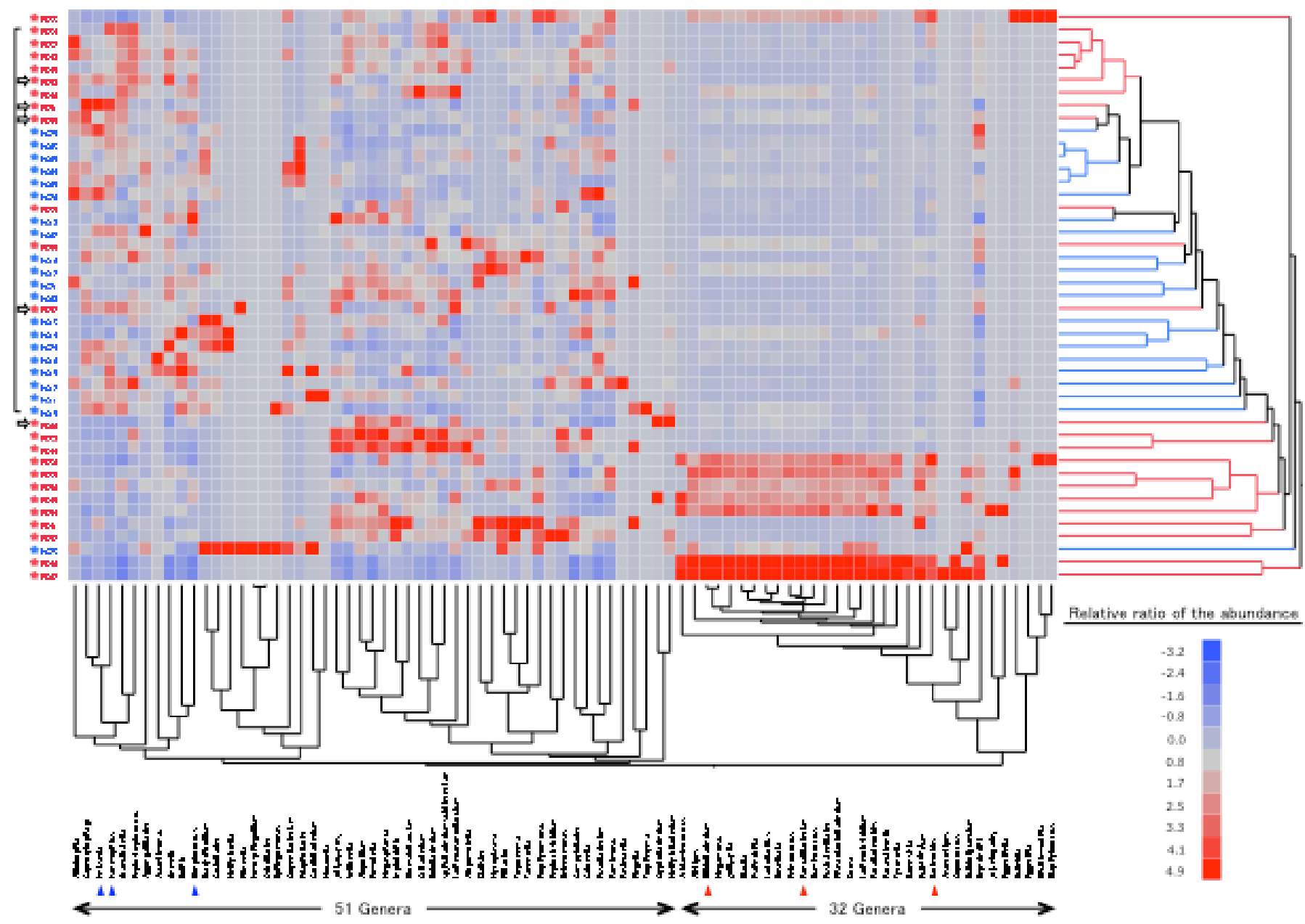

Figure 3 Interindividual bacterial compositional variation at the genus level. The samples from 45 subjects and 83 dominant genera are represented on a double-hierarchical clustering heat map. The blue and red squares represent lower and higher abundances, respectively. The clusters on the right side indicate the similarity among individual profiles at the genus level. The clusters at the bottom indicate the genera showing similarity in the frequency of identification among samples. Cited with permission from ref ${ }^{35}$. 
this study, those results concerning the genera strongly suggested that commensal intestinal bacteria often reflux into the stomach in patients with FD.

While the link between the change in the GF microbiota and the pathophysiology of FD remains to be clarified, it is noteworthy that the abundance of the genus Escherichia (included in the 32 genera) in the FD group was significantly greater than in the HC group, whose per cent ratios were $1.6 \pm 2.1$ and $0.3 \pm 0.3($ mean $\pm S D$, $\mathrm{p}<0.05)$, respectively. This is because lipopolysaccharides (LPS) are major cell components of Escherichia and capable of stimulating leucocytes to generate proinflammatory cytokines. Low-grade duodenal and/or gastric antral inflammation has been observed and proposed as an important pathophysiological mechanism in patients with FD. ${ }^{36}$ Vanheel $e t a l^{37}$ reported that patients with FD showed increased duodenal mucosal permeability that might potentially lead to mucosal inflammation. Given that LPS and bile acids induce acceleration of mucosal permeability, the inflammation in the duodenal/antral region might be caused by those toxic substances in patients with FD.

The treatment of patients with FD with LG21 restored the dysbiotic microbiota to that found in HC volunteers. The dominance of Bacteroidetes over Proteobacteria was markedly weakened after the LG21 treatment (figure 2). Indeed, the number of subjects whose Bacteroidetes to Proteobacteria ratio decreased after the treatment was as many as 18 out of 24 patients. In addition, the prevalence of intestinal-type genera, including Escherichia, was significantly decreased in patients with FD after this treatment (unpublished data). Therefore, probiotics appear effective in the treatment of FD through the normalisation of gastric microbiota, particularly via the reduction of Escherichia.

\section{FUTURE ASPECT}

In contrast to intestinal microbiota, the role of gastric microbiota in human health and physiology has been ignored so far, as the bacterial load in the stomach is considered too small to exert a significant influence on the stomach and the downstream intestine. Now, we would like to emphasise that it will be necessary to fully understand the pathophysiological influence of gastric microbiota on them, because the number of persons who continuously take efficient antiacid drugs such as PPI and potassium-competitive acid blocker is recently increasing so much in the world. In their stomach, the size of gastric microbiota mass is dramatically increased so that a great number of gastric bacteria migrate to the intestine. It still remains unclear what effect such migrant gastric bacteria exert on intestinal microbiota and the host. If it is pathogenic, probiotics will be needed to improve such dysbiotic gastric microbiota, in addition to their usage for $\mathrm{H}$. pylori infection and FD as already mentioned in this article.

Contributors YK wrote the outline for the Introduction, Gastric microbiota and Future aspect sections, and is responsible for the overall content as the guarantor.
TO wrote the section on Use for functional dyspepsia. KK wrote the sections on Development of a probiotic Lactobacillus strain for the suppression of $H$. pylori in human and on Gastric microbial community associated with the occurrence of gastric cancers. YA constructed the ground design of this article and wrote the section on Biological role of indigenous gastric microbiota.

Funding The authors have not declared a specific grant for this research from any funding agency in the public, commercial or not-for-profit sectors.

\section{Competing interests None declared.}

Patient consent for publication Nor required.

Provenance and peer review Not commissioned; externally peer reviewed.

Data availability statement There are no data in this work. No data are available.

Open access This is an open access article distributed in accordance with the Creative Commons Attribution Non Commercial (CC BY-NC 4.0) license, which permits others to distribute, remix, adapt, build upon this work non-commercially, and license their derivative works on different terms, provided the original work is properly cited, appropriate credit is given, any changes made indicated, and the use is non-commercial. See: http://creativecommons.org/licenses/by-nc/4.0/.

\section{REFERENCES}

1. Fuller R. Probiotics in man and animals. J Appl Bacteriol 1989;66:365-78.

2. World Health Organization. Fao of the United nations and who: health and nutritional properties of probiotics in food including powdered milk with live lactic acid bacteria. 2001

3. Hill C, Guarner F, Reid G, et al. The International scientific association for probiotics and prebiotics consensus statement on the scope and appropriate use of the term probiotic. Nat Rev Gastroenterol Hepatol 2014;11:506-14.

4. Qin J, Li R, Raes J, et al. A human gut microbial gene catalogue established by metagenomic sequencing. Nature 2010;464:59-65.

5. Malfertheiner P, Megraud F, O'Morain C, et al. Current concepts in the management of Helicobacter pylori infection-The Maastricht 2-2000 consensus report. Aliment Pharmacol Ther 2002;16:167-80.

6. Delgado S, Cabrera-Rubio R, Mira A, et al. Microbiological survey of the human gastric ecosystem using culturing and pyrosequencing methods. Microb Ecol 2013;65:763-72.

7. Bik EM, Eckburg PB, Gill SR, et al. Molecular analysis of the bacterial microbiota in the human stomach. Proc Natl Acad Sci U S A 2006;103:732-7.

8. Li X-X, Wong GL-H, To K-F, et al. Bacterial microbiota profiling in gastritis without Helicobacter pylori infection or non-steroidal antiinflammatory drug use. PLoS One 2009;4:e7985.

9. Tsuda A, Suda W, Morita H, et al. Influence of proton-pump inhibitors on the luminal microbiota in the gastrointestinal tract. Clin Transl Gastroenterol 2015;6:e89.

10. Suerbaum S, Michetti P. Helicobacter pylori infection. N Engl J Med 2002;347:1175-86

11. Eun CS, Kim BK, Han DS, et al. Differences in gastric mucosal microbiota profiling in patients with chronic gastritis, intestinal metaplasia, and gastric cancer using pyrosequencing methods. Helicobacter 2014;19:407-16.

12. Stockbruegger RW. Bacterial overgrowth as a consequence of reduced gastric acidity. Scand J Gastroenterol 1985;20:7-15.

13. Ruddell WS, Axon AT, Findlay JM, et al. Effect of cimetidine on the gastric bacterial flora. Lancet 1980;1:672.

14. Wilder-Smith $\mathrm{CH}$, Spirig $\mathrm{C}$, Krech $\mathrm{T}$, et al. Bactericidal factors in gastric juice. Eur J Gastroenterol Hepatol 1992;4:885-91.

15. Kabir AM, Aiba Y, Takagi A, et al. Prevention of Helicobacter pylori infection by lactobacilli in a gnotobiotic murine model. Gut 1997;41:49-55.

16. Takahashi $\mathrm{H}$, Nakano $\mathrm{Y}$, Matsuoka $\mathrm{T}$, et al. Role of Indigenous lactobacilli in gastrin-mediated acid production in the mouse stomach. Appl Environ Microbiol 2011;77:6964-71.

17. Schubert ML, Peura DA. Control of gastric acid secretion in health and disease. Gastroenterology 2008;134:1842-60.

18. Stanghellini V, Tosetti C, Paternicò A, et al. Risk indicators of delayed gastric emptying of solids in patients with functional dyspepsia. Gastroenterology 1996;110:1036-42.

19. Saito $Y$, Suzuki H, Tsugawa $H$, et al. Dysfunctional gastric emptying with down-regulation of muscle-specific microRNAs in Helicobacter pylori-infected mice. Gastroenterology 2011;140:189-98.

20. Sasaki Y. Investigation of acid-resistance mechanisms of an anti-H. pylori strain of Lactobacillus gasseri using a DNA microarray technique. Bioscience and Industry 2004;62:17-20. 
21. Sakamoto I, Igarashi M, Kimura K, et al. Suppressive effect of Lactobacillus gasseri OLL 2716 (LG21) on Helicobacter pylori infection in humans. J Antimicrob Chemother 2001;47:709-10.

22. Gotteland M, Brunser O, Cruchet S. Systematic review: are probiotics useful in controlling gastric colonization by Helicobacter pylori? Aliment Pharmacol Ther 2006;23:1077-86.

23. Boonyaritichaikij S, Kuwabara K, Nagano J, et al. Long-term Administration of Probiotics to Asymptomatic Pre-school Children for Either the Eradication or the Prevention of Helicobacter pylori Infection. Helicobacter 2009;14:202-7.

24. Tong JL, Ran ZH, Shen J, et al. Meta-Analysis: the effect of supplementation with probiotics on eradication rates and adverse events during Helicobacter pylori eradication therapy. Aliment Pharmacol Ther 2007;25:155-68.

25. Deguchi R, Nakaminami H, Rimbara E, et al. Effect of pretreatment with Lactobacillus gasseri OLL2716 on first-line Helicobacter pylori eradication therapy. J Gastroenterol Hepatol 2012;27:888-92.

26. Ferreira RM, Pereira-Marques J, Pinto-Ribeiro I, et al. Gastric microbial community profiling reveals a dysbiotic cancer-associated microbiota. Gut 2018;67:226-36.

27. Lambert J, Hull R. Upper gastrointestinal tract disease and probiotics. Asia Pacific J Clin Nutr 1996;5:31-5.

28. Enck P, Azpiroz F, Boeckxstaens G, et al. Functional dyspepsia. Nat Rev Dis Primers 2017;3:1-19.

29. Tack J, Bisschops R, Sarnelli G. Pathophysiology and treatment of functional dyspepsia. Gastroenterology 2004;127:1239-55.

30. Miwa H, Hirai S, Nagahara A, et al. Cure of Helicobacter pylori infection does not improve symptoms in non-ulcer dyspepsia patients-a double-blind placebo-controlled study. Aliment Pharmacol Ther 2000;14:317-24.

31. Tan VPY, Liu KSH, Lam FYF, et al. Randomised clinical trial: rifaximin versus placebo for the treatment of functional dyspepsia. Aliment Pharmacol Ther 2017;45:767-76.

32. Takagi A, Yanagi $\mathrm{H}$, Ozawa $\mathrm{H}$, et al. Effects of Lactobacillus gasseri OLL2716 on Helicobacter pylori-associated dyspepsia: a multicenter randomized double-blind controlled trial. Gastoenterol Res Practice 2016.

33. Ohtsu T, Takagi A, Uemura N, et al. The ameliorating effect of Lactobacillus gasseri OLL2716 on functional dyspepsia in Helicobacter pylori-uninfected individuals: a randomized controlled study. Digestion 2017;96:92-102.

34. Matsueda K, Hongo M, Tack J, et al. Clinical trial: dose-dependent therapeutic efficacy of acotiamide hydrochloride (Z-338) in patients with functional dyspepsia - $100 \mathrm{mg}$ t.i.d. is an optimal dosage. Neurogastroenterol Motil 2010;22:e173:618-e173.

35. Igarashi M, Nakae H, Matsuoka T, et al. Alteration in the gastric microbiota and its restoration by probiotics in patients with functional dyspepsia. BMJ Open Gastroenterol 2017;3 :e000144.

36. Talley NJ, Walker MM, Aro P, et al. Non-Ulcer dyspepsia and duodenal eosinophilia: an adult endoscopic population-based casecontrol study. Clin Gastroenterol Hepatol 2007;5:1175-83.

37. Vanheel H, Vicario M, Vanuytsel T, et al. Impaired duodenal mucosal integrity and low-grade inflammation in functional dyspepsia. Gut 2014;63:262-71. 